\title{
Risk factors of uncontrolled symptoms using the standard dose of second-generation H1 -antihistamines in chronic spontaneous urticaria children
}

\author{
Vanlaya Koosakulchai, Araya Yuenyongviwat, Pasuree Sangsupawanich
}

\begin{abstract}
Background: A standard dose of second-generation H1 -antihistamines is recommended as the first-line treatment of chronic spontaneous urticaria (CSU), previous studies have found that approximately 20-50\% of CSU children fail to control their symptoms and required step-up treatments.
\end{abstract}

Objective: To evaluate the predictors of uncontrolled symptoms with first-line medication and describe the treatment outcomes of CSU children in the southern region of Thailand.

Methods: This retrospective chart review of CSU patients, aged 2-18 years, who were initially treated with the standard dose of second-generation H1 -antihistamine at the Pediatric Allergy Clinic, Songkhlanagarind Hospital, from January 2008 to July 2018. The data were collected at the initial visit (demographic data, onset of rash, frequency of urticaria, presence of angioedema, previous resolved CU, laboratory investigation results) and follow-up visits (treatment outcome, time to controlled urticaria).

Results: The medical records of 192 CSU children were reviewed; their median age were 8.5 years and the mean frequency of rash was 4 days/week. Forty-seven children (24.4\%) fail to controlled symptoms with a standard dose of second-generation $\mathrm{H} 1$-antihistamines and a factor significantly associated was frequency of rash for more than 4 days per week $(\mathrm{OR}=4.36, P<0.001)$. The median time to controlled urticaria was 1.28 months.

Conclusions: Most of CSU children in the southern region of Thailand experienced controlled symptoms with a standard dose of second-generation H1 -antihistamines, and the frequency of urticaria for more than 4 days per week was a factor associated with uncontrolled symptoms that regimen.

Key words: chronic spontaneous urticaria, child, second-generation H1 -antihistamines, uncontrolled symptoms, risk factor

\section{Citation:}

Koosakulchai, V., Yuenyongviwat, A., Sangsupawanich, P. (2022). Risk factors of uncontrolled symptoms using the standard dose of second-generation $\mathrm{H} 1$-antihistamines in chronic spontaneous urticaria children. Asian Pac J Allergy Immunol, 40(2), 121-125. https://doi.org/10.12932/ap-030619-0573

\section{Affiliation:}

Division of Allergy and Immunology, Department of Pediatrics, Faculty of Medicine, Prince of Songkla University, Hat Yai, Songkhla, Thailand

\section{Corresponding author:}

Vanlaya Koosakulchai

Division of Allergy and Immunology, Department of Pediatrics

Faculty of Medicine, Prince of Songkla University

15 Karnjanavanich Road, Hat Yai, Songkhla 90110, Thailand

E-mail: windyfernny@gmail.com

\section{Introduction}

Chronic urticaria (CU) entails the presentation of urticarial rash at least twice a week for longer than 6 weeks. ${ }^{1}$ $\mathrm{CU}$ is classified as chronic spontaneous urticaria (CSU) and inducible urticaria; CSU constitutes the majority of cases of CU. ${ }^{2-3}$ In the United Kingdom, the prevalence of CSU children has been reported at $0.1-0.3 \%,{ }^{4}$ while the prevalence of CSU children in Thailand is $13 \% .^{5}$ CSU significantly affects 
the quality of life of children and is associated with socioeconomic burden. ${ }^{6}$ There are several guidelines for the management of CSU; a standard dose of second-generation $\mathrm{H1}$-antihistamines is recommended as the first-line treatment in all of the guidelines. If the patient's symptoms cannot be controlled within 2-4 weeks, the options are up-dosing the second-generation $\mathrm{H} 1$-antihistamine by 2-4 folds, adding another second-generation $\mathrm{H} 1$-antihistamine, adding a first-generation antihistamine at bedtime, adding an $\mathrm{H} 2$ antihistamine or adding a leukotriene receptor antagonist. For cases resistant to these treatments, adding cyclosporine A or omalizumab is indicated..$^{-9}$

Previous studies have found that approximately $20-50 \%$ of children and $70-80 \%$ of adult CSU patients fail to control their symptoms with a standard dose of second-generation $\mathrm{H} 1$ -antihistamine. ${ }^{10-13}$ Some studies have reported an association between the presence of angioedema, drug allergy and family history of CSU and uncontrolled symptoms with the standard dose of antihistamine. ${ }^{10-11}$ However, there are limited data about predictors and treatment outcomes among children in Thailand, who cannot control their symptoms with a standard dose of second-generation $\mathrm{H} 1$-antihistamines. The aim of this study was to evaluate the predictors of uncontrolled symptoms with a standard dose of second-generation H1 -antihistamines and describe the treatment outcomes of CSU children in the southern region of Thailand.

\section{Methods \\ Study design and population}

The medical records of CSU patients, aged 2-18 years, who were diagnosed with chronic spontaneous urticaria and initially treated with the standard dose of second-generation $\mathrm{H} 1$-antihistamine (cetirizine, loratadine, levocetirizine, desloratadine, fexofenadine) at the Pediatric Allergy Clinic, Songkhlanagarind Hospital, from January 2008 to July 2018, were reviewed. The patients who had underlying diseases related to urticaria or angioedema such as maculopapular cutaneous mastocytosis, urticarial vasculitis, bradykinin-mediated angioedema or cryopyrin-associated periodic syndrome, and patients on immunosuppressive drugs were excluded.

We calculated the sample size necessary to detect the risk factors of uncontrolled symptoms with a standard dose of second-generation $\mathrm{H} 1$-antihistamines using the formula for testing two independent proportions (P1 - the proportion of patients who had angioedema $=0.45$; P2 - the proportion of patients who did not have angioedema $=0.12, \mathrm{n} 2 / \mathrm{n} 1$ = 1). ${ }^{11}$ The results indicated a required sample of at least 34 per group. Additionally, we calculated the sample size to detect the rate of uncontrolled symptoms with a standard dose second-generation $\mathrm{H} 1$-antihistamines using the formula for estimating the infinite population proportion ( $\mathrm{P}$; rate of uncontrolled symptoms with a standard antihistamine dose $=$ 0.185). ${ }^{12}$ It was found that the overall sample size needed to be at least 232 .

\section{Data review from medical records}

The data were collected from the medical records at the initial visit consisted of demographic information (sex, age, weight, height, drug allergy, current medications, past history of atopy, and family history of atopy), onset of rash, frequency of urticaria (days/week), presence of angioedema, previous resolved CU, laboratory investigation results (CBC, ESR, ANA, anti-dsDNA, thyroid function test, skin prink test or sIgE to aeroallergens) and type of second-generation $\mathrm{H} 1$-antihistamine drug. The data from medical records at follow-up visits were consisted of treatment outcome and time to controlled urticaria. The treatment outcome of the standard dose of second-generation $\mathrm{H} 1$-antihistamines was evaluated 2-4 weeks after the start of treatment, and uncontrolled symptoms were defined as the presence of urticaria at least 2 days/week while on the same daily dose of antihistamine.

\section{Statistical analysis}

The data were recorded using Epidata and analyzed using the $\mathrm{R}$ statistical software. The demographic data are presented as mean \pm standard deviation or median (IQR) for continuous data, and number (\%) for categorical data. The rate of uncontrolled symptoms was presented as number (\%) for categorical data. The predictors for uncontrolled symptoms were analyzed using the chi-square and Fisher's exact tests for sample proportion comparisons and the T-test and Mann-Whitney U Test to compare the sample mean/medians. The strength of association was measured by means of odds ratios (OR) with 95\% confidence intervals (95\% CI). A $P$ value of less than 0.05 was regarded as statistically significant. The categorical data concerning medications used to control urticaria are presented as number (\%), while the time to control urticaria was tested by using the time-to-event analysis.

\section{Ethics statement}

This study was approved by the Human Research Committee of the Faculty of Medicine, Prince of Songkla University, Thailand.

\section{Results \\ Demographic data}

The medical records of 192 CSU children were reviewed; their median age were 8.5 years (Table 1 ). The proportions of males and females were equal. The medium duration of urticaria before diagnosis was 4.5 months, and the mean frequency of urticaria was 4 days/week. Just under half of the children (46.9\%) had angioedema. Seventy-two children (37.5\%) had a personal history of atopy, and asthma was the most common comorbidity. Approximately, one-fourth of the children had a family history of atopy. The laboratory investigations illustrated an absolute eosinophilic count (AEC) mean of 195 cell/ $\mathrm{mm}^{3}$. Moreover, an abnormal ESR (> $20 \mathrm{~mm} / \mathrm{h}$ ) was found in 31 children (21.4\%) and a positive ANA in 13 children (9\%); however, the anti-ds DNA test resulted negative in all of them and none of the children had autoimmune disease. Aeroallergen sensitization was evaluated in 113 children, and just under half of them $(49.4 \%)$ had positive results. The thyroid function test was performed on 114 children. However, while 6 children had abnormal results as subclinical hypothyroid, none of the patient had autoimmune thyroiditis. 
Table 1. Baseline characteristics

\begin{tabular}{|c|c|}
\hline Characteristic & $\begin{array}{c}\text { Total } \\
(\mathrm{N}=192)\end{array}$ \\
\hline Age of diagnosis (years), mean (SD) & $8.5(3.7)$ \\
\hline Sex, male, n (\%) & $96(50.0)$ \\
\hline Weight $(\mathrm{kg})$, mean $(\mathrm{SD})$ & $31.3(15.4)$ \\
\hline Height (cm), mean (SD) & $128(23.2)$ \\
\hline $\begin{array}{l}\text { Duration of urticaria before diagnosis (months), median } \\
\text { (IQR) }\end{array}$ & $4.5(0.8,87)$ \\
\hline Frequency of urticaria (days/week), mean (SD) & $4.3(2.0)$ \\
\hline Presence of angioedema, $\mathrm{n}(\%)$ & $90(46.9)$ \\
\hline Previous resolved CU, n (\%) & $4(2.1)$ \\
\hline Personal history of atopy, $\mathrm{n}(\%)$ & $72(37.5)$ \\
\hline - Asthma & $32(16.7)$ \\
\hline - Allergic rhinitis & $28(14.6)$ \\
\hline - Atopic dermatitis & $4(2.1)$ \\
\hline - Food allergy & $22(11.5)$ \\
\hline Drug allergy, n (\%) & $19(9.9)$ \\
\hline Family history of atopy, $\mathrm{n}(\%)$ & $47(24.5)$ \\
\hline Family history of CU, n (\%) & $13(6.8)$ \\
\hline \multicolumn{2}{|l|}{ Laboratory investigation } \\
\hline - AEC (cell/mm³), median (IQR) & $195(0,1414)$ \\
\hline - ESR abnormal, n (\%) & $31(20.4)$ \\
\hline - ANA positive, $\mathrm{n}(\%)$ & $13(9)$ \\
\hline - Anti-ds DNA positive, n (\%) & $0(0.0)$ \\
\hline - Thyroid function test abnormal, $\mathrm{n}(\%)$ & $6(5.3)$ \\
\hline - Aeroallergen sensitization & $39(49.4)$ \\
\hline
\end{tabular}

Abbreviation: SD, Standard deviation; IQR, Interquartile range; CU, Chronic urticaria; AEC, Absolute eosinophil count; ESR, Erythrocyte sedimentation rate; ANA, Antinuclear antibody; Anti-ds DNA, Anti-double stranded DNA antibody
Treatment outcome of chronic spontaneous urticaria in children

All of the children were initially treated with a standard dose of second-generation $\mathrm{H} 1$-antihistamines, which comprised cetirizine $(52.6 \%)$, loratadine $(40.6 \%)$, desloratadine (4.7\%), fexofenadine (1.6\%) and levocetirizine $(0.5 \%)$. The mean follow-up duration after the initial treatment with a standard dose of second-generation $\mathrm{H} 1$-antihistamines was 1 month. The prevalence of controlled symptoms with a standard dose of second-generation $\mathrm{H} 1$-antihistamines was $75.6 \%$. Forty-seven children whose symptoms were uncontrolled symptoms received step-up treatment, and five children were lost to follow up. Thirty-five children (18.2\%) had their symptoms controlled with a double dose of second-generation H1- antihistamines. Six children achieved controlled symptoms with higher doses of antihistamine; a three-fold dosed in five persons and a four-fold doses in one person. Otherwise, one person's urticaria symptoms were controlled with a standard dose of second-generation H1- antihistamines combined with a dose of first-generation $\mathrm{H} 1$-antihistamines at bedtime and an $\mathrm{H} 2$-antihistamine.

Overall, the median time to controlled urticaria was 1.28 months (1.08 months in the patients who experienced controlled symptoms with a standard dose of second-generation $\mathrm{H} 1$-antihistamines and 2.83 months in the patients who failed to have their symptoms controlled with this medication).

Risk factors for uncontrolled symptoms with a standard dose of second-generation H1- antihistamines in chronic spontaneous urticaria patients

The rate of uncontrolled symptoms with a standard dose of second-generation $\mathrm{H} 1$ - antihistamines was $24.4 \%$ and the factors that might lead to uncontrolled symptoms are shown in Table 2. A factor significantly associated with uncontrolled symptoms with a standard dose of second-generation $\mathrm{H} 1$-antihistamines was frequency of rash for more than 4 days per week $(P<0.01)$.

However, the other risk factors such as presence of angioedema, previous resolved CU, personal or family history of atopy, drug allergy, and all of the laboratory investigation findings were not found to associate with uncontrolled symptoms with this step of treatment.

Table 2. Risk factors associated with uncontrolled symptoms with a standard dose of second-generation H1 -antihistamines in CSU patients

\begin{tabular}{|c|c|c|c|}
\hline Characteristic & $\begin{array}{l}\text { Uncontrolled } \\
\text { with standard dose } \mathrm{AH} \\
(\mathrm{N}=47)\end{array}$ & $\begin{array}{c}\text { Controlled } \\
\text { with standard dose } \mathrm{AH} \\
(\mathrm{N}=145)\end{array}$ & $P$ value \\
\hline Sex, male, n (\%) & $24(51.1)$ & $71(50.0)$ & 1.00 \\
\hline Age of onset of CSU (years), median (IQR) & $8.9(3.6,11.8)$ & $7.7(5,10.6)$ & 0.67 \\
\hline Frequency of urticaria, n (\%) & & & $<0.01$ \\
\hline$\leq 4$ days/week & $13(27.7)$ & $80(56.3)$ & \\
\hline$>4$ days/week & $34(72.3)$ & $62(43.7)$ & \\
\hline
\end{tabular}


Table 2. (Continued)

\begin{tabular}{|c|c|c|c|}
\hline Characteristic & $\begin{array}{l}\text { Uncontrolled } \\
\text { with standard dose } \mathrm{AH} \\
\qquad(\mathrm{N}=47)\end{array}$ & $\begin{array}{l}\text { Controlled } \\
\text { with standard dose } \mathrm{AH} \\
(\mathrm{N}=145)\end{array}$ & $P$ value \\
\hline Presence of angioedema, $\mathrm{n}(\%)$ & $25(53.2)$ & $64(45.1)$ & 0.42 \\
\hline Previous resolved CU, n (\%) & $1(2.1)$ & $3(2.1)$ & 1.00 \\
\hline Personal history of atopy, n (\%) & $19(40.4)$ & $53(37.3)$ & 0.84 \\
\hline Drug allergy, n (\%) & $7(14.9)$ & $12(8.5)$ & 0.26 \\
\hline Family history of atopy, n (\%) & $13(27.7)$ & $32(22.5)$ & 0.60 \\
\hline Family history of CU, n (\%) & $3(6.4)$ & $9(6.3)$ & 1.00 \\
\hline \multicolumn{4}{|l|}{ Laboratory investigation } \\
\hline AEC (cell/mm³), median (IQR) & $179(101,285)$ & $224(138,399)$ & 0.08 \\
\hline ESR abnormal, n (\%) & $3(7.9)$ & $28(24.6)$ & 0.05 \\
\hline ANA positive, $\mathrm{n}(\%)$ & $5(13.5)$ & $8(7.5)$ & 0.32 \\
\hline Aeroallergen sensitization, $\mathrm{n}(\%)$ & $8(42.1)$ & $31(51.7)$ & 0.64 \\
\hline Thyroid function test abnormal, $\mathrm{n}(\%)$ & $0(0)$ & $6(7.4)$ & 0.18 \\
\hline
\end{tabular}

Abbreviation: AH, Antihistamine; CSU, Chronic spontaneous urticaria; CU, Chronic urticaria; AEC, Absolute eosinophil count; ESR, Erythrocyte sedimentation rate; ANA, Antinuclear antibody

\section{Multivariate analysis}

The multivariate analysis findings of the factors associated with uncontrolled symptoms with a standard dose of second -generation $\mathrm{H} 1$-antihistamines are shown in Table 3. A significant association was found between; uncontrolled symptoms and a frequency of rash for more than 4 days per week $(\mathrm{OR}=4.67 ; 95 \%$ CI $1.8-0.4, P \leq 0.001)$.

Table 3. Multivariate logistic analysis of factors associated with uncontrolled symptoms with a standard dose of second -generation $\mathrm{H1}$-antihistamines in CSU patients

\begin{tabular}{|lcc|} 
& \multicolumn{2}{c|}{ Multivariated analysis } \\
\cline { 2 - 3 } & ORa $(\mathbf{9 5} \% \mathrm{CI})$ & P value \\
\hline Frequency of urticaria $>$ 4 days/week & $4.36(1.8,10.4)$ & $<0.001$ \\
\hline Presence of angioedema & $1.03(0.4,2.5)$ & 0.95 \\
\hline Previous resolved CU & $0.89(0.1,11.9)$ & 0.93 \\
\hline Personal history of atopy & $0.65(0.2,2.15)$ & 0.48 \\
\hline Drug allergy & $1.13(0.3,4.2)$ & 0.86 \\
\hline Family history of CU & $0.61(0.05,7.8)$ & 0.70 \\
\hline Laboratory investigation & $0.99(0.99,1.00)$ & 0.28 \\
\hline AEC, median (IQR) & $0.62(0.09,4.5)$ & 0.64 \\
\hline ESR abnormal & & \\
\hline
\end{tabular}

Abbreviation: ORa, Adjusted odds ratio; CI, Confidence interval; CSU, Chronic spontaneous urticaria; CU, Chronic urticaria; AEC, Absolute eosinophil count; ESR, Erythrocyte sedimentation rate

\section{Discussion}

This cohort study on 192 children with CSU showed a rate of $24.4 \%$ for uncontrolled symptoms with a standard dose of second-generation $\mathrm{H} 1$-antihistamines and a risk factor associated with uncontrolled symptoms with this step of treatment was frequency of rash for more than 4 days per week. However, the other risk factors, namely the presence of angioedema, previous resolved CU, personal or family history of atopy, drug allergy and all of laboratory investigation findings, did not reveal any association with uncontrolled symptoms with a standard dose of second-generation $\mathrm{H} 1$-antihistamines. Almost all of the children achieved controlled symptoms with an up-dosing to a double dose of a second-generation $\mathrm{H} 1$-antihistamine drug, and the overall medium time to controlled symptoms was 1.28 months.

According to the guidelines for the management of CSU, a standard dose of second-generation $\mathrm{H1}$ - antihistamines is recommended as the first-line treatment. If the patient's symptoms are not controlled within 2-4 weeks, a step-up treatment is indicated. In Thailand, Chansakulporn et al., ${ }^{12}$ performed a prospective study among 94 CSU children between 2003 and 2009 at Siriraj Hospital, and the results indicated that $18.5 \%$ of the patients did not have their symptoms controlled with a standard dose of antihistamines. This was similar to our study's finding we found that $24.4 \%$ of our participants did not respond to the initial treatment. However, our proportion was lower than that of the study by Lee et al., ${ }^{10}$ who performed an observational prospective study in Singapore in $98 \mathrm{CSU}$ children and found that $50 \%$ of the patients failed to control their symptoms with a standard antihistamine dose. This might be explained by the difference in the patients' severity of urticaria at baseline because this study enrolled patients who had daily or almost daily urticaria symptoms for at least 6 weeks, while the medium frequency of urticaria 
in our study was 4 days. When comparing our study with studies in adults, Marin-Cabanas et al., ${ }^{11}$ who performed a cross-sectional study on 100 CSU adults, found $82 \%$ incidence of uncontrolled symptoms with a standard dose of antihistamine. Such a high rate of uncontrolled symptoms with the initial treatment in this study compared to our study might owe to the fact that this study had a higher rate of chronic autoimmune urticaria (positive ANA 12\%, positive antithyroid antibody 20\%) than our study (positive ANA 9\%).

Furthermore, previous studies have evaluated the risk factors associated with uncontrolled symptoms with a standard dose of second-generation $\mathrm{H} 1$-antihistamines. Lee et al., ${ }^{10}$ found that angioedema, drug allergy and family history of CSU were associated with uncontrolled symptoms. Likewise, Marin-Cabanas et al., ${ }^{11}$ who performed a cross-sectional study among $100 \mathrm{CSU}$ adults, reported that the presence of angioedema was associated with a lack of response to treatment with a standard antihistamine dose. In Thailand, the data concerning the risk factors for uncontrolled symptoms with a standard dose of antihistamine are limited. In our study, we did not find any significant association between angioedema, drug allergy and family history of CSU and uncontrolled urticaria symptoms in childhood.

According to our study, only the frequency of urticaria for more than 4 days per week was an associating factor. The activity scales that have been proposed to integrate symptom intensity and its impact on daily life such as the Urticaria Severity Score (USS) consist of 9 questions, and the number of days of urticaria is one of them. This indicates that the frequency of urticaria is associated with the severity of the disease, and it might affect the response to the initial treatment.

Based on the results of our study, if the patent has urticaria more than 4 days/week for at least 6 weeks, the treatment should involve more than a standard dose of second-generation of $\mathrm{H} 1$-antihistamines in order to control the patient's symptoms early. However, we need further studies to confirm this hypothesis.

The strengths of this study lie with its large sample size and low rate of loss to follow-up. There were some limitations in this study that are worth mentioning. Firstly, we did not evaluate disease severity using UAS scores. However, we employed an indirect assessment of the disease severity by evaluating the frequency of rash and the patient's symptoms. Secondly, we did not perform ASST, which is used in the diagnosis of CAU; however, this test is not usually carried out in real practice because it is difficult to perform, and it does not change the treatment. Finally, we enrolled only patients who received a standard dose of second-generation $\mathrm{H} 1$-antihistamines as an initial treatment. This might have introduced a selection bias since we did not enroll patients with a more severe disease that were referred to our hospital due to the initial treatment failure.

In conclusion, most of our CSU patients experienced controlled symptoms with a standard dose of second-generation $\mathrm{H} 1$-antihistamines, and the frequency of urticaria for more than 4 days per week was a factor associated with uncontrolled symptoms that regimen.

\section{Acknowledgements}

The authors gratefully acknowledge all of the Epidemiology staff from the Faculty of Medicine, Prince of Songkla University for assistance about the data analysis.

\section{Conflict of interest declaration}

The authors declare no potential conflicts of interest with respect to the research, authorship, and/or publication of this article.

\section{References}

1. Grattan CE, Sabroe RA, Greaves MW. Chronic urticaria. J Am Acad Dermatol. 2002;46:645-57.

2. Zuberbier $T$, Aberer W, Asero R, Abdul Latiff AH, Baker D, Ballmer-Weber $\mathrm{B}$, et al. The EAACI/GA ${ }^{2} \mathrm{LEN} / \mathrm{EDF} / \mathrm{WAO}$ guideline for the definition, classification, diagnosis and management of urticaria. Allergy. 2018;73:1393-414.

3. Zuberbier T, Balke M, Worm M, Edenharter G, Maurer M. Epidemiology of urticaria: a representative cross-sectional population survey. Clin Exp Dermatol. 2010;35:869-73.

4. Khakoo G, Sofianou-Katsoulis A, Perkin MR, Lack G. Clinical features and natural history of physical urticaria in children. Pediatr Allergy Immunol. 2008;19:363-6.

5. Tuchinda M, Srimaruta N, Habanananda S, Vareenil J, Assatherawatts A. Urticaria in Thai children. Asian Pac J Allergy Immunol. 1986;4:41-5.

6. O'Donnell BF. Urticaria: impact on quality of life and economic cost. Immunol Allergy Clin North Am. 2014;34:89-104.

7. Bernstein JA, Lang DM, Khan DA, Craig T, Dreyfus D, Hsieh F, et al. The diagnosis and management of acute and chronic urticaria: 2014 update. J Allergy Clin Immunol. 2014;133:1270-7.

8. Powell RJ, Leech SC, Till S, Huber PA, Nasser SM, Clark AT. BSACI guideline for the management of chronic urticaria and angioedema. Clin Exp Allergy. 2015;45:547-65.

9. Zuberbier $\mathrm{T}$, Aberer $\mathrm{W}$, Asero R, Bindslev-Jensen C, Brzoza Z, Canonica GW, et al. The EAACI/GA(2) LEN/EDF/WAO Guideline for the definition, classification, diagnosis, and management of urticaria: the 2013 revision and update. Allergy. 2014;69:868-87.

10. Lee XH, Ong LX, Cheong JY, Sultana R, Rao R, Lim HH, et al. A stepwise approach in the management of chronic spontaneous urticaria in children. Asia Pac Allergy. 2016;6:16-28.

11. Marín-Cabañas I, Berbegal-de Gracia L, de León-Marrero F, Hispán P, Silvestre JF. Management of Chronic Spontaneous Urticaria in Routine Clinical Practice Following the EAACI/GA(2)LEN/EDF/WAO Guidelines. Actas Dermosifiliogr. 2017;108:346-53.

12. Chansakulporn S, Pongpreuksa S, Sangacharoenkit P, Pacharn P, Visitsunthorn N, Vichyanond P, et al. The natural history of chronic urticaria in childhood: a prospective study. J Am Acad Dermatol. 2014; 71:663-8.

13. Chantbuddhiwet S, Ruxrungtham K, Korkij W, Noppakun N. The Natural History of Chronic Urticaria: A Cross-sectional Retrospective Study at a Tertiary Care Center, Bangkok, Thailand. J Allergy Clin Immunol. 2009;123:S107.

14. Curto-Barredo L, Archilla LR, Vives GR, Pujol RM, Gimenez-Arnau AM. Clinical Features of Chronic Spontaneous Urticaria that Predict Disease Prognosis and Refractoriness to Standard Treatment. Acta Derm Venereol. 2018;98:641-7. 